Arq. Bras. Med. Vet. Zootec., v.67, n.6, p.1572-1580, 2015

\title{
Avaliação dos valores de troponina I, eletrocardiograma e ecocardiograma em felinos sedados com cetamina e midazolam, suplementados ou não com oxigênio
}

\author{
[Troponin I, electrocardiography and echocardiography values in felines sedated with ketamine \\ and midazolam, supplemented or not with oxygen] \\ R. Tocheto ${ }^{1}$, V.S. Padilha ${ }^{1}$, H.M. Cardoso ${ }^{1}$, E.H. Bitencourt ${ }^{2}$, J. Volpato ${ }^{1}$, \\ J.N.B. Andrade ${ }^{3}$, N. Oleskovicz ${ }^{1}$ \\ ${ }^{1}$ Programa de pós-graduação do Centro de Ciências Agroveterinárias - CAV/UDESC - Lages, SC \\ ${ }^{2}$ Escola de Veterinária - Universidade Federal de Minas Gerais - Belo Horizonte, MG \\ ${ }^{3}$ Médico veterinário autônomo - Curitiba, PR
}

\section{RESUMO}

Lesões no miocárdio, causadas por baixa perfusão e oxigenação cardíaca, podem ser ocasionadas por fármacos anestésicos, como a cetamina. Essas lesões podem ser identificadas por meio de biomarcadores específicos e, dentre estes, destaca-se a troponina I. O objetivo deste estudo foi avaliar as alterações cardiovasculares com base nos valores de troponina I (TnI), eletrocardiograma (ECG) e ecocardiograma em gatos sedados com cetamina e midazolam, suplementados ou não com oxigênio. Utilizaram-se 12 gatos machos, hígidos, nos quais se avaliaram os valores de troponina I, eletro e ecocardiografia, frequência cardíaca (FC) e pressão arterial sistólica (PAS) no momento basal (M0). Na sequência, os animais foram sedados com a associação de $10 \mathrm{mg} \cdot \mathrm{kg}^{-1}$ de cetamina e $0,5 \mathrm{mg} \cdot \mathrm{kg}^{-1}$ de midazolam pela via intramuscular. Decorridos aproximadamente 10 minutos, os animais foram alocados aleatoriamente em dois grupos: com e sem suplementação de oxigênio via máscara facial (GCO e GSO, respectivamente), sendo submetidos novamente aos exames citados. Foram coletadas amostras sanguíneas, para dosagem de TnI em seis, 12 e 24 horas após a administração dos agentes anestésicos. Não foram observadas alterações significativas na FC, na PAS e no ECG após a administração dos tratamentos em ambos os grupos. Os valores médios de TnI elevaram-se significativamente em T6 quando comparados ao basal em ambos os grupos, com médias de $0,507 \pm 0,335 \mathrm{ng} / \mathrm{mL}$ no GSO e $0,777 \pm 0,505 \mathrm{ng} / \mathrm{mL}$ no GCO. Na ecocardiografia, o débito cardíaco (DC) reduziu em M1 em ambos os grupos, quando comparados aos valores basais, sendo M0 0,472 $\pm 0,115$ e M1 0,234 $\pm 0,08$ no GSO e M0 0,356 $\pm 0,095$ e M1 0,222 $\pm 0,09$ no $\mathrm{GCO}$, expressos em L/min. Conclui-se que a administração de cetamina e midazolam em gatos hígidos não promove alterações eletrocardiográficas, aumenta os valores de troponina I, com pico de seis horas após a administração, reduz o débito cardíaco, e que a suplementação de oxigênio $100 \%$ via máscara facial não atenua tais alterações.

Palavras-chave: biomarcadores, gatos, miocárdio, cetamina

\begin{abstract}
Myocardium injuries caused by low myocardial oxygenation and perfusion might be induced by anesthetics agents like ketamine. These injuries can be detected by specific biomarkers and, among them, troponin I. The aim of this study was to evaluate the cardiovascular changes based on troponin I (TnI) values, electrocardiography (ECG) and echocardiography in cats sedated with ketamine and midazolam, supplemented or not with oxygen. Blood samples were collected from 12 intact male healthy cats for troponin I (TO) and they were then submitted to electrocardiographic and echocardiographic evaluation, as well as measurements of heart rate (HR) and systolic blood pressure (SBP) (MO). Subsequently, they were ketamine-midazolam (10mg. $\mathrm{kg}^{-1}$ and $0,5 \mathrm{mg} \cdot \mathrm{kg}^{-1}$ respectively) anesthetized by intramuscular route. After about 10 minutes, the animals were randomly allocated into two groups with or without oxygen
\end{abstract}

Recebido em 5 de fevereiro de 2015

Aceito em 17 de agosto de 2015

E-mail: nisetochetto@yahoo.com.br 
supplementation (GCO or GSO, respectively), again being subjected to the tests mentioned. Blood samples for troponin I were collected at 6, 12 and 24 hours after sedation. HR, SBP and ECG did not change among groups. The TnI values rise significantly in T6 comparing to baselines in both groups $(0,507 \pm 0,335 \mathrm{ng} / \mathrm{mL}$ in $\mathrm{GSO}$ and $0,777 \pm 0,505 \mathrm{ng} / \mathrm{mL}$ in GCO). In echocardiography, the cardiac output decreased at M1, in both groups compared to baseline (M0 0,472 $\pm 0,115$ and M1 0,234 $\pm 0,08$ in GSO and MO 0,356 $\pm 0,095$ and 0,222 $\pm 0,09$ in $G C O, L / m i n)$. We concluded that ketamine and midazolam sedation in healthy cats did not cause changes electrocardiography, increase troponin I values, with an 6 hours peak after administration, reduces cardiac output and oxygen supplementation, via facial mask, did not attenuated these alterations.

Keywords: biomarkers, cats, myocardium, ketamine

\section{INTRODUÇÃO}

O sistema cardiovascular é constantemente exposto a injúrias causadas por anestésicos inalatórios ou injetáveis, os quais atuam sobre o fluxo sanguíneo coronariano e podem produzir efeitos diretos e indiretos sobre a pressão de perfusão coronariana, o tempo de perfusão e o tônus vascular coronário, liberando troponina I das células miocárdicas (Slack, 2011). Essas alterações podem levar à redução da perfusão tecidual e à oxigenação miocárdica e, como consequência, à lesão nos miócitos (Fonfara et al., 2010). De longa data, a cetamina é utilizada na anestesia de pequenos animais para realização de procedimentos cirúrgicos de curta duração. Em associação com outros agentes na indução anestésica, esse fármaco tem como principal característica a ocorrência de efeitos simpatomiméticos (Riviere e Papich, 2009), além de promover aumento do transporte e do consumo de oxigênio pelo miocárdio (Lin, 2013).

A identificação de alterações cardíacas em animais assintomáticos por meio de testes bioquímicos permite diagnosticar cardiopatias com maior acurácia e em menor tempo (Sisson, 2002). A troponina cardíaca (TnI) foi descrita como um marcador altamente específico e sensível para detectar danos miocárdicos em muitas espécies de mamíferos, e sua estrutura é muito similar entre elas (Yonezawa et al., 2010).

Devido à escassa literatura e ao amplo uso da associação de cetamina e midazolam na rotina anestésica veterinária, este estudo objetivou avaliar o impacto e a ocorrência de alterações sobre o miocárdio, com base na dosagem sérica da troponina I e nos parâmetros eletro e ecocardiográficos desse protocolo, em felinos hígidos, suplementados ou não com oxigênio via máscara facial.

\section{MATERIAL E MÉTODOS}

Este estudo foi aprovado pelo Comitê de Ética e Bem-Estar Animal (CETEA) da instituição de origem (protocolo n॰ 1.19.12). Foram utilizados 12 gatos adultos, machos, com idade variando entre sete meses e quatro anos, sem raça definida, com peso médio de $4,1 \pm 0,6 \mathrm{~kg}$, comprovadamente hígidos por meio de exame clínico e exames laboratoriais (perfil renal [creatinina e ureia] e hepático [alanina aminotransferase, fosfatase alcalina], proteína sérica total, albumina, globulina e glicose). Os animais foram incluídos no estudo somente após o consentimento prévio dos proprietários e eram provenientes da rotina clínica do Hospital de Clínica Veterinária, sendo admitidos para realização de orquiectomia eletiva.

Previamente ao início do estudo, os animais foram alojados, durante dois dias, em uma sala silenciosa de $8 \mathrm{~m}^{2}$, a qual dispunha de um espaço suficiente para interação do avaliador com o animal e de uma gaiola de metal $\left(0,8 \mathrm{~m}^{3}\right)$, para adaptação ao local, durante o qual receberam alimentação à base de ração comercial e água $a d$ libitum.

Após a adaptação dos animais, foram coletados os dados basais (M0) de pressão arterial sistólica (PAS); frequência cardíaca (FC); eletrocardiograma (ECG) e ecocardiograma (exame completo, sendo apenas os valores referentes à determinação do débito cardíaco incluídos e analisados neste estudo) e troponina I (T0). Inicialmente, avaliou-se a PAS utilizandose Doppler vascular portátil (Doppler 841-A, Parks Medical Eletronics ${ }^{\circledR}$, Inc. Aloha, Oregon, USA) e FC, mediante auscultação torácica com 
estetoscópio convencional. Em seguida, realizouse a ecocardiografia bidimensional e em modo $\mathrm{M}$ e Doppler pulsátil, contínuo e colorido, com probe de $5 \mathrm{MHz}$ (HD15 Ultrasound System Philips ${ }^{\circledR}$ Healthcare). Com o animal em decúbito lateral direito, posicionando-se o transdutor na janela ecocardiográfica paraesternal direita, localizada entre o terceiro e sexto espaços intercostais (EIC) direitos, entre o esterno e a articulação costocondral, foram obtidas as imagens do eixo longitudinal e do eixo transversal e realizadas as medidas ecocardiográficas. A partir do eixo-longo, foram obtidas as visões quatro-câmaras e trato de saída do ventrículo esquerdo, e, quando no eixo-curto, foram observados os cortes nos níveis apical, papilar, cordal, mitral e aórtico.

As imagens foram inicialmente obtidas em modo $\mathrm{B}$, avaliando-se as relações anatômicas entre as estruturas, a contratilidade, os aspectos morfofuncionais valvares, a presença de jatos de regurgitação com o Doppler colorido e o gradiente de pressão da valva pulmonar. Foram medidos o átrio esquerdo e a aorta e calculada a relação átrio esquerdo/aorta. $\mathrm{O}$ eixo curto serviu de orientação para a obtenção das imagens em modo $\mathrm{M}$, com exposição simultânea do modo bidimensional na tela. Com a linha do cursor sobre o nível das cordas tendíneas, obtiveram-se as medidas do diâmetro interno do ventrículo esquerdo ao final da diástole (DIVEd) e da sístole (DIVEs). A partir dessas medidas, obtevese a fração de encurtamento (SF).

Pela janela paraesternal caudal esquerda (apical), em modo bidimensional, foram observadas as relações anatômicas entre as estruturas cardíacas, os aspectos morfofuncionais valvares, a contratilidade miocárdica e calculou-se o gradiente de pressão da valva aórtica. $\mathrm{O}$ débito cardíaco e a FE (fração de ejeção) foram calculados pelo método de Simpson modificado, conforme descrito por Boon (2011).

Em seguida, coletaram-se-se aproximadamente 3 - $5 \mathrm{~mL}$ de sangue venoso da veia jugular, para mensuração da concentração sérica basal de troponina I (T0), armazenando em tubos com ativador de coágulo (Tubo Gel BD SST® II Advance $\left.{ }^{\circledR}\right)$. A amostra coletada foi centrifugada a $1500 \mathrm{~g}$, durante oito minutos, para separação do soro. O material centrifugado foi divido em duplicatas, armazenado em Eppendorfs, identificados e, imediatamente, congelados a $20^{\circ} \mathrm{C}$.

Vinte e quatro horas após (M1), os animais foram submetidos a jejum alimentar de 12 horas e hídrico de duas horas e receberam a associação de $0,5 \mathrm{mg} \cdot \mathrm{kg}^{-1}$ de midazolam (Dormire $\AA$, $5 \mathrm{mg} / \mathrm{mL}$, Cristália Produtos Químicos e Farmacêuticos, Itapira/SP, Brasil) e $10 \mathrm{mg} \cdot \mathrm{kg}^{-1}$ de cetamina (Vetaset ${ }^{\circledR}$, $100 \mathrm{mg} / \mathrm{mL}$, Ford Dodge Saúde Animal, São Paulo, Brasil), pela via intramuscular, sendo posteriormente alocados aleatoriamente em dois grupos: $\mathrm{GCO}(n=6)$, com suplementação de oxigênio $(1 \mathrm{~L} / \mathrm{min})$ por meio de máscara facial, durante todo $\mathrm{o}$ período da sedação, e GSO $(n=6)$, sem suplementação de oxigênio. $\mathrm{O}$ tempo de sedação foi considerado a partir do momento em que o animal adotava o decúbito lateral até o retorno ao decúbito esternal.

A partir do momento em que os animais permitiram ou permaneceram em decúbito lateral, novamente a PAS e os parâmetros ecocardiográficos foram avaliados (M1), conforme descrito anteriormente. Em ato contínuo, realizou-se também a eletrocardiografia digital (Eletrocardiógrafo Digital TEB ${ }^{\circledR}$ ), utilizando-se a derivação II, com velocidade de $50 \mathrm{~mm} /$ segundo e amplitude de $2 \mathrm{~N}$ (M1), que contemplava a frequência cardíaca, o ritmo e a medida de ondas, os intervalos e os segmentos. Com os animais ainda sedados, realizou-se tricotomia na região cervical ventral para posterior introdução, de maneira asséptica, de um catéter venoso central (Intracath ${ }^{\circledR}$ 19G BD) em uma das veias jugulares, conectado a um adaptador PRN, o qual foi devidamente fixado e protegido por gaze cirúrgica e bandagem elástica e, então, utilizado para coletas sanguíneas para mensuração da concentração de troponina I (cTnI) em seis, 12 e 24 horas (T6; T12 e T24, respectivamente) após a administração dos tratamentos. No dia seguinte, após a última coleta de sangue para dosagem da cTnI, foi realizada a orquiectomia eletiva dos animais, não sendo incluído esse procedimento durante $\mathrm{o}$ período de estudo.

Ao término do estudo, todas as amostras (soro) foram armazenadas em uma caixa térmica com gelo seco e enviadas ao Laboratório Maricondi São Carlos/SP, para processamento e dosagem de troponina I, por meio do método de 
quimioluminescência, com valor mínimo de detecção de $0,060 \mathrm{ng} / \mathrm{mL}$.

\section{RESULTADOS E DISCUSSÃO}

Mesmo com amplo uso de longa data dos anestésicos dissociativos em medicina veterinária, não foram encontrados, até o presente momento, estudos que comprovem as alterações cardiovasculares causadas pelo uso da associação de cetamina e midazolam em gatos, nas doses propostas, sem a presença de outras variáveis, como a realização de procedimentos cirúrgicos. Os escassos estudos existentes avaliaram o uso da cetamina associada a outros agentes anestésicos (opioides, alfa-2-agonistas e tranquilizantes) procurando determinar alterações cardiovasculares e/ou a facilidade de manipulação do paciente, objetivando mínima influência nos resultados de exames complementares, como, por exemplo, a ecocardiografia (Cilli et al., 2010; Slack et al., 2011; Ward et al., 2012).

O peso médio dos animais do GCO e GSO foi de $3,8 \pm 0,5 \mathrm{~kg}$ e $4,3 \pm 0,6 \mathrm{~kg}$, respectivamente, não havendo diferença significativa entre grupos. Também não houve diferença estatística significativa entre grupos para os valores basais de hemograma e bioquímica sérica.

A cetamina é um fármaco que tem como característica a estimulação simpática, levando ao aumento da frequência cardíaca e da pressão arterial (Lin, 2013). No entanto, no presente estudo, os valores de pressão arterial sistólica e de frequência cardíaca não diferiram significativamente dos valores basais dentro de cada grupo nem entre grupos ou após a administração do protocolo anestésico (Tab. 1).

Tabela 1. Valores médios e desvio-padrão da pressão arterial sistólica (PAS), frequência cardíaca (FC), DC (débito cardíaco) (método de Simpson modificado); VDF (volume diastólico final); DIVEd (diâmetro interno do ventrículo esquerdo ao final da diástole) e FE (fração de ejeção) nos momentos basal e durante a sedação com cetamina e midazolam, em gatos hígidos suplementados (GCO) ou não com oxigênio (GSO)

\begin{tabular}{lllll} 
& $\begin{array}{l}\text { GSO } \\
\text { basal }\end{array}$ & $\begin{array}{l}\text { GCO } \\
\text { basal }\end{array}$ & $\begin{array}{l}\text { GSO } \\
\text { sedação }\end{array}$ & $\begin{array}{l}\text { GCO } \\
\text { sedação }\end{array}$ \\
\hline PAS (mmHg) & $131 \pm 18$ & $135 \pm 14$ & $161 \pm 46$ & $142 \pm 38$ \\
FC (bpm) & $182 \pm 29$ & $181 \pm 36$ & $210 \pm 40$ & $176 \pm 50$ \\
DC (L/min) & $0,472 \pm 0,105 \mathrm{a}$ & $0,356 \pm 0,095 \mathrm{~b}$ & $0,234 \pm 0,08 \mathrm{~A}$ & $0,222 \pm 0,09 \mathrm{~A}$ \\
VDF (mL) & $2,84 \pm 0,62$ & $2,53 \pm 0,72$ & $1,75 \pm 0,51 \mathrm{~A}$ & $1,92 \pm 0,74 \mathrm{~A}$ \\
DIVEd (cm) & $1,70 \pm 0,12 \mathrm{a}$ & $1,52 \pm 0,16 \mathrm{~b}$ & $1,51 \pm 0,19$ & $1,41 \pm 0,21$ \\
FE $(\%)$ & $83,66 \pm 5,64$ & $83,65 \pm 6,22$ & $65,85 \pm 14,66 \mathrm{~A}$ & $73,56 \pm 8,44 \mathrm{~A}$ \\
\hline
\end{tabular}

Fonte: próprio autor. Letras maiúsculas indicam diferença estatística significativa quando comparado ao basal (M0), ANOVA RM seguido pelo teste SNK $(\mathrm{P}<0,05)$. Letras minúsculas indicam diferença significativa entre grupos, teste $t$ $(\mathrm{P}<0,05)$.

Os valores obtidos de FC e PAS no momento basal estavam acima dos valores esperados para a espécie, mesmo com a realização de ambientação prévia. Nesse sentido, sabe-se que o estresse leva à liberação de catecolaminas, culminando com o aumento da FC e da PAS. Diante disso, sugere-se que o estresse causado pela contenção física e pela mudança de ambiente foi, muito provavelmente, o responsável pela elevação dos valores basais, mascarando os efeitos da cetamina sobre a frequência cardíaca e a pressão arterial sistólica ao longo do tempo de avaliação. Esses achados corroboram o encontrado por Quimby et al.
(2011), que avaliaram as alterações nos parâmetros fisiológicos de gatos examinados em hospitais veterinários e em seu domicílio, constatando valores significativamente maiores de FC e PAS nos animais que foram avaliados no hospital.

Em relação aos valores eletrocardiográficos como: onda $\mathrm{P}(\mathrm{mV})$, onda $\mathrm{R}(\mathrm{mV})$, onda $\mathrm{T}(\mathrm{mV})$, onda $\mathrm{Q}(\mathrm{mV})$, onda $\mathrm{P}(\mathrm{ms})$, intervalo PR (ms), intervalo QRS (ms) e intervalo QT (ms), não foram observadas diferenças significativas entre momentos e entre grupos, conforme descrito na Tab. 2. 
Tabela 2. Valores médios e desvio-padrão dos valores de frequência cardíaca $(\mathrm{FC})$, onda $\mathrm{P}(\mathrm{mV})$, onda $\mathrm{R}$ $(\mathrm{mV})$, onda $\mathrm{T}(\mathrm{mV})$, onda $\mathrm{Q}(\mathrm{mV})$, onda $\mathrm{P}(\mathrm{ms})$, intervalo PR $(\mathrm{ms})$, intervalo QRS $(\mathrm{ms})$ e intervalo QT (ms) do eletrocardiograma nos momentos basal (M0) e após a sedação com cetamina e midazolam (M1) em gatos hígidos, suplementados (GCO) ou não com oxigênio (GSO)

\begin{tabular}{lllllllll}
\hline ECG basal $(\mathrm{M} 0)$ & & & & & \\
Variáveis & $\mathrm{P}(\mathrm{mV})$ & $\mathrm{R}(\mathrm{mV})$ & $\mathrm{T}(\mathrm{mV})$ & $\mathrm{Q}(\mathrm{mV})$ & $\mathrm{P}(\mathrm{ms})$ & $\mathrm{PR}(\mathrm{ms})$ & QRS $(\mathrm{ms})$ & QT $(\mathrm{ms})$ \\
\hline GSO & $0,14 \pm 0,04$ & $0,45 \pm 0,23$ & $0,11 \pm 0,06$ & - & $37,11 \pm 11,06$ & $67,44 \pm 14,77$ & $43,11 \pm 5,08$ & $122,11 \pm 40,32$ \\
GCO & $0,14 \pm 0,03$ & $0,30 \pm 0,07$ & $0,15 \pm 0,03$ & $0,02 \pm 0,05$ & $45,88 \pm 14,50$ & $79,12 \pm 11,59$ & $41,75 \pm 11,78$ & $148,37 \pm 22,25$ \\
\hline Variáveis & $\mathrm{P}(\mathrm{mV})$ & $\mathrm{R}(\mathrm{mV})$ & $\mathrm{T}(\mathrm{mV})$ & $\mathrm{Q}(\mathrm{mV})$ & $\mathrm{P}(\mathrm{ms})$ & $\mathrm{PR}(\mathrm{ms})$ & $\mathrm{QRS}(\mathrm{ms})$ & $\mathrm{QT}(\mathrm{ms})$ \\
\hline ECG M1 & & & & & & & $39,89 \pm 6,75$ & $142,22 \pm 29,27$ \\
\hline GSO & $0,13 \pm 0,02$ & $0,41 \pm 0,18$ & $0,14 \pm 0,07$ & $0,05 \pm 0,11$ & $35,44 \pm 7,57$ & $70,22 \pm 8,18$ & 39,89 \\
GCO & $0,12 \pm 0,03$ & $0,30 \pm 0,12$ & $0,10 \pm 0,04$ & $0,02 \pm 0,07$ & $35,75 \pm 11,78$ & $74,00 \pm 17,50$ & $42,50 \pm 7,21$ & $141,62 \pm 30,71$ \\
\hline
\end{tabular}

Fonte: próprio autor.

Os valores do ECG após a administração da cetamina, em ambos os grupos, permaneceram dentro dos valores de referência para a espécie. Conforme descrito por Tilley (1992), a presença de alterações eletrocardiográficas, como o supra ou infradesnivelamento do segmento ST, pode indicar má oxigenação do miocárdio. Porém, Gennaro et al. (2008) destacam que as alterações do segmento ST nem sempre são evidentes quando na presença de isquemia miocárdica. Apesar de terem sido identificadas alterações nos valores de troponina I neste estudo, em ambos os grupos, e estas indicarem a ocorrência de hipóxia miocárdica, não foram detectadas alterações eletrocardiográficas que poderiam sugerir essa injúria, conforme citado anteriormente por Gennaro et al. (2008). Ainda, em outro estudo realizado por Souza et al. (2002), que avaliaram eletrocardiogramas de cães anestesiados com cetamina racêmica e $\mathrm{S}(+)$ isoladas, na dose de $20 \mathrm{mg} \cdot \mathrm{kg}^{-1} \mathrm{IM}$, não foram encontradas alterações significativas no ECG, fato esse que reforça os resultados obtidos no presente estudo.

Entretanto, Hravnak et al. (2009) avaliaram pacientes humanos com aneurisma subaracnoideo por hemorragia e correlacionaram o prolongamento do segmento QT (mediante avaliação com Holter) com os níveis de troponina I, demonstrando que não somente o prolongamento desse segmento foi maior nos pacientes com maiores níveis de troponina, como também essas alterações eletrocardiográficas persistiram, em $40 \%$ dos pacientes avaliados, por até 48 horas após a avaliação basal. Esse resultado sugere, que a troponina I é um biomarcador mais específico e precoce do que as alterações de um eletrocardiograma. No entanto, provavelmente, alterações eletrocardiográficas poderiam ter sido diagnosticadas no presente estudo se o tempo de avaliação eletrocardiográfica tivesse sido maior. Mas, devido ao estresse e à dificuldade da realização do exame eletrocardiográfico em felinos acordados, a avaliação tardia não foi executada.

Observaram-se mínimas alterações na maioria dos parâmetros ecocardiográficos avaliados, exceto no DC (débito cardíaco). Esses resultados corroboram os encontrados por Ward et al. (2012), que utilizaram dois protocolos de sedação: acepromazina e butorfanol, associados ou não à cetamina, para a realização de ecocardiograma em gatos e não identificaram alterações ecocardiográficas importantes em grande parte dos parâmetro avaliados.

Foram observados valores menores de DC no GCO em relação ao GSO, no momento basal, e houve redução significativa do DC após a administração de cetamina/midazolam, em ambos os grupos, quando comparados aos valores basais $(37,7 \%$ e $50,4 \%$, respectivamente), conforme a Tab. 1 .

O DC é definido pelo volume de sangue bombeado pelo ventrículo esquerdo a cada minuto, podendo ser determinado, portanto, pela seguinte fórmula: $\mathrm{DC}=\mathrm{VSxFC}$, em que $\mathrm{VS}$ representa o volume sistólico e FC a frequência cardíaca (Gayton e Hall, 2002). Ainda segundo Mohrman e Heller (1997), o débito cardíaco está sujeito a alterações, de forma diretamente proporcional à pressão arterial (PA) e inversamente proporcional à resistência vascular 
total (RVT), como expressa a fórmula: DC = PA/RVT. Portanto, o débito cardíaco pode modificar-se em decorrência de alterações na pré-carga, como volume sanguíneo, pressão intrapleural, tônus venoso e componente atrial; na contratilidade e na pós-carga, como resistência periférica total e volume residual no sistema venoso. No presente estudo, as alterações na pré-carga e na contratilidade foram descartadas, visto que o volume sistólico foi mantido e alterações no componente atrial, como fibrilação atrial, não foram observadas no eletrocardiograma. Assim, sugere-se que as reduções observadas no DC foram decorrentes de alterações na pós-carga. Porém, por dificuldades técnicas, como impossibilidade de cateterização arterial em felinos acordados e a baixa confiabilidade de aferição das pressões arterial sistólica, média e diastólica pelo método oscilométrico, os parâmetros de resistência periférica total não foram mensurados. Brown et al. (1997) sugerem que o aumento da resistência vascular total tem como consequência o aumento no volume residual de sangue venoso, resultando em um menor retorno venoso, fato que explicaria os valores reduzidos do volume diastólico final (VDF) e do diâmetro interno do ventrículo esquerdo ao final da diástole (DIVEd) póstratamento, no presente estudo (Tab. 2). Os valores médios do DIVEd foram menores no GCO quando comparados ao GSO no momento basal. Essas diferenças também podem ser explicadas pela variação de peso entre os grupos, já que o peso médio do grupo GSO foi clinicamente maior que o peso do GCO, embora sem diferença estatisticamente significativa.

Como não houve redução da $\mathrm{FC}$ entre os grupos dentro do mesmo momento, sugere-se que a redução do DC ocorreu devido à queda dos valores de VDF, o qual está diretamente relacionado com o relaxamento cardíaco durante a diástole. Houve redução do VDF em M1 quando comparado ao M0 para ambos os grupos (Tab. 1). De acordo com o descrito por Lin (2013), após a administração de cetamina, ocorre um aumento do consumo de oxigênio pelo miocárdio. No presente estudo, foi observado que o grupo que recebeu suplementação com oxigênio teve queda do DC menor quando comparado ao grupo que não foi suplementado, sugerindo, dessa forma, que a maior oferta de oxigênio por meio de máscara facial pode melhorar o trabalho cardíaco. Observou-se redução significativa nos valores da fração de ejeção (FE) no momento pós-sedação, quando comparado ao basal, para ambos os grupos (Tab. 1). Visto que esse parâmetro reflete o volume ejetado do ventrículo esquerdo durante a sístole ventricular, havendo, desse modo, um decréscimo no VDF, espera-se também uma queda da FE, conforme descrito por Boon (2011). Ainda, segundo Zaugg et al. (2004), em casos em que a FC está aumentada, poderá ocorrer aumento do trabalho cardíaco e, consequentemente, aumento da demanda de oxigênio pelo miocárdio, ocorrendo redução do volume de ejeção, redução do débito cardíaco e, subsequentemente, redução da perfusão coronariana.

Os valores da troponina I (TnI) basais, em ambos os grupos, apresentaram-se dentro dos limites considerados fisiológicos para a espécie felina (Tab. 3), corroborando valores descritos por Sleeper et al. (2001) que podem variar entre $0,03-0,16 \mathrm{ng} / \mathrm{mL}$. Nesse contexto, sabe-se que as troponinas cardíacas possuem alta sensibilidade e especificidade para o músculo cardíaco e são consideradas os biomarcadores de eleição para detecção de injúria no miocárdio (Wells e Sleeper, 2008). De acordo com Hagman et al. (2007), qualquer dano nos miócitos resulta em perda da integridade celular, provocando a liberação de troponina I na circulação. Desse modo, a concentração sérica dessa proteína está correlacionada com a gravidade da lesão miocárdica.

Os valores de troponina I (Tab. 3) obtidos no presente estudo tiveram um comportamento semelhante aos descritos na literatura, em que o pico da liberação da $\mathrm{TnI}$ pode ser observado em seis horas após a aplicação da cetamina e do midazolam, sugerindo que o início da liberação ocorreu logo após o uso desses fármacos. Esses valores mantiveram-se acima dos de referência até T12, demonstrando queda após o pico em seis horas. No momento 24 horas, a média dos valores da TnI já havia retornado aos valores fisiológicos para a espécie em ambos os grupos, podendo ser este um indicativo de que o dano causado pela cetamina e pelo midazolam, nas doses preconizadas neste estudo, foi transitório. O'brien et al. (2006) e Wells e Sleeper (2008) determinaram que o aumento dos níveis da concentração da troponina I (cTnI) começam a ocorrer em torno de duas horas após a lesão nos 
miócitos, atingindo o pico após 12 a 24 horas. Já de acordo com Babuin e Jaffe (2005), a TnI é detectada na corrente sanguínea entre quatro e oito horas após a lesão, podendo permanecer por um período de três a sete dias. Conforme relatado por O'brien et al. (2006), Wells e Sleeper (2008) e Langhorn et al. (2013), o aumento persistente da troponina I pode sugerir danos irreversíveis ou lesão ativa nas células miocárdicas. Esse fato, provavelmente, não ocorreu no presente estudo devido à redução gradual que ocorreu nos níveis de TnI após seis horas da anestesia.

Tabela 3. Valores médios e desvio-padrão da concentração sérica de troponina I $(\mathrm{ng} / \mathrm{mL})$ em gatos hígidos, anestesiados com cetamina e midazolam, suplementados (GCO) ou não com oxigênio (GSO)

\begin{tabular}{lcc} 
Momentos & \multicolumn{2}{c}{ Concentração troponina I $(\mathrm{ng} / \mathrm{mL})$} \\
\hline T0 & GS0 & GC0 \\
T6 & $0,082 \pm 0,035$ & $0,060 \pm 0,000$ \\
T12 & $0,342 \pm 0,279$ & $0,282 \pm 0,116$ \\
T24 & $0,155 \pm 0,114$ & $0,077 \pm 0,042$ \\
\hline
\end{tabular}

Letras maiúsculas indicam diferença estatística significativa quando comparado ao basal (M0), ANOVA RM seguido pelo teste SNK $(\mathrm{P} \leq 0,05)$. T0, T6, T12 e T24 representam, respectivamente, momento basal, seis, 12 e 24 horas após a sedação.

Alguns protocolos sedativos ou anestésicos podem resultar em alterações cardiovasculares significativas, levando à depressão desse sistema, o que ocasiona hipotensão e redução da perfusão tecidual, além da redução da oxigenação do miocárdio e morte celular (Cilli et al., 2010). No presente estudo, observou-se aumento significativo nos níveis da TnI no momento T6 em $100 \%$ dos animais de ambos os grupos em relação aos valores basais. Já em T12 e T24 os valores médios da troponina $\mathrm{I}$ em ambos os grupos foram reduzindo sem apresentar diferença estatística em relação ao momento basal. Esses dados diferem dos descritos em cães por Cilli et al. (2010), que detectaram aumento dos níveis séricos da TnI em 14\% dos cães saudáveis (ASA I e II) submetidos a vários protocolos anestésicos, para diferentes procedimentos cirúrgicos. Saunders et al. (2009) também observaram aumento dos valores de TnI em 10\% dos cães após procedimentos cirúrgicos, concomitante à ocorrência de eventos intraoperatórios, como hemorragias, períodos de hipóxia $\left(\mathrm{SpO}_{2}<92 \%\right)$, extremos de $\mathrm{FC}$ e $\mathrm{PA}$, e sugerem que esses eventos podem ter contribuído para o aumento da concentração da TnI. No presente estudo, acredita-se que ocorreram apenas eventos relacionados à sedação, como, por exemplo, valores altos de PAS e queda do DC em ambos os grupos, o que pode ter contribuído para a alteração dos valores da troponina I, já que nenhum procedimento cirúrgico foi realizado durante a coleta de dados. A introdução do catéter central também poderia ser um dos fatores que levaram ao aumento dos valores de troponina I, conforme citado em um estudo realizado por Shih et al. (2009) em cães que passaram por procedimentos de cateterização cardíaca e tiveram aumento dos valores de cTnI. Acredita-se que, no presente estudo, é improvável que tenha ocorrido lesão miocárdica pelo uso do catéter, pois este não atingiu nenhuma estrutura cardíaca, devido ao seu comprimento reduzido. Outro fator que pode explicar a grande diferença entre as porcentagens de aumento dos valores de troponina I deste estudo (100\%) comparado aos estudos citados anteriormente (14 e 10\%) é que, no presente estudo, os momentos de coleta das amostras foram em maior número e em menor intervalo a zero, seis, 12 e 24 horas, ao contrário dos demais estudos, que coletaram somente basal e 24 horas após o estímulo anestésico/cirúrgico. Assim, pode-se verificar que o pico dos valores de cTnI ocorreu em seis horas, retornando, na maioria dos animais, aos valores basais dentro de 24 horas. Isto pode sugerir que, nos estudos realizados por Cilli et al. (2010) e Saunders et al. (2009), o aumento da troponina I possa ter ocorrido, retornando aos valores basais em até 24 horas, porém sem ser detectado.

A mensuração da saturação do oxigênio da hemoglobina não foi realizada, devido principalmente à dificuldade da manutenção do sensor por tempo suficiente para a detecção dos valores nos momentos basais, visto que os animais encontravam-se acordados. Esse fato foi uma limitação do estudo, pois, sem esses dados, não se pode afirmar se houve ou não períodos de hipóxia durante a sedação. Os animais foram alocados em dois grupos, com e sem suplementação de oxigênio (GCO e GSO), pois 
esperava-se que o grupo que recebeu a suplementação durante o período transanestésico apresentasse valores menores de TnI, conforme afirmam Gunnewiek e Van Der Hoeven (2004), que, além da taquicardia, uma insuficiente suplementação de oxigênio pode contribuir para lesão no miocárdio. No entanto, não houve diferença significativa entre os animais suplementados ou não com oxigênio com relação aos valores de troponina I, sugerindo, dessa forma, que o possível efeito benéfico da suplementação de oxigênio não impediu a liberação de troponina I na circulação desses gatos. A possível ocorrência de hipóxia após a administração dos anestésicos neste estudo também pode ser explicada pela administração da cetamina, já que esse fármaco, conforme citado por Gennaro et al. (2008), pode levar a um aumento na frequência cardíaca, devido à estimulação simpática e, dessa forma, a um aumento na demanda de oxigênio, bem como a uma redução no seu fornecimento ao miocárdio, além de promover vasoespasmo coronariano. Ainda, esses mesmos autores relatam que o dano cardíaco pode ser isquêmico, mas não ser devido a alguma doença coronariana, pois é sabido que, muitas vezes, ocorre aumento do estresse da parede ventricular, o que causa um desequilíbrio entre a oferta e a demanda de oxigênio para os miócitos.

As principais limitações encontradas neste estudo foram a dificuldade para a realização dos exames ecocardiográficos e para a mensuração da pressão arterial no momento basal com os animais acordados, mesmo após a ambientação deles, além da não aferição dos valores da saturação da oxi-hemoglobina durante o período de sedação.

Sugere-se, como estudo futuro, que a avaliação dos valores de TnI seja realizada conjuntamente com a de gases sanguíneos, para determinar se realmente existe correlação entre o aumento dos níveis séricos de troponina I com menores níveis de saturação da oxi-hemoglobina.

\section{CONCLUSÕES}

O uso de cetamina e midazolam em gatos hígidos não altera os parâmetros eletrocardiográficos, mas promove aumento da concentração sérica de troponina I, com pico de seis horas após a administração, e reduz o débito cardíaco. A suplementação de oxigênio $100 \%$ via máscara facial não atenua tais alterações.

\section{REFERÊNCIAS}

BABUIN, L; JAFFE, A.S. Troponin: the biomarker of choice for the detection of cardiac injury. Can. Lead. Med. J., v.173, p.1191-1202, 2005.

BOON, J.A. Veterinary echocardiography. 2. ed. Oxford: Wiley-Blackwell, 2011. 480p.

BROWN, H.; KOZLOWSKI, R.D. Phisiology and pharmacology of the heart. Oxford: Blackwell Science, 1997. 643p.

CILLI, F.; ALIBHAI, I.; ARMITAGE-CHAN, E. et al. Incidence of elevation of cardiac troponina I prior to and following routine general anaethesia in dogs. Vet. Anaesth. Analg., v.37, p.409-416, 2010.

FONFARA, S.; LOUREIRO, S.; SWIFT, S. et al. Cardiac troponin I as a marker for severity and prognosic of cardiac disease in dogs. Vet. J., v.184, p.334-339, 2010.

GENNARO, L.; BRUNETTI, N.D.; CUCULO, A. et al. Increased Troponin levels in nonischemic cardiac conditions and noncardiac diseases. J. Interv. Cardiol., v.21, p.129-139, 2008.

GUNNEWIEK, J.; VAN DER HOEVEN, J. Cardiac troponin elevations among critically ill patients. Curr. Opin. Crit. care., v.10, p.342-346, 2004.

GAYTON, C.A.; HALL, J.E. Fisiologia médica. 10.ed. Rio de Janeiro: Guanabara Koogan, 2002. $973 \mathrm{p}$.

HAGMAN, R.; LAGERSTEDT, A.S.; FRANSSON, B.A. et al. Cardiac troponina I levels in canine piometra. Acta Vet. Scand., v.49, p.6, 2007.

HRAVNAK, M.; FRANGISKAKIS, M.; CRAGO, E.A. et al. Elevated cardiac troponin I and relationship to persistence of electrocardiographic and echocardiographic abnormalities after aneurysmal subarachnoid hemorrhage. J. Am. Health Assoc., v.40, p.34783484, 2009. 
LANGHORN, R.; WILLESEN, J.L.; TARNOW, I.; KJELGAARD-HANSEN, M. Evaluation of a high-sensitivity assay for measurement of canine and feline serum cardiac troponin I. Vet. Clin. Pathol., v.42, p.490-498, 2013.

LIN, H. Dissociative anesthetics. In: TRANQUILLI, W.J.; THURMON, J.C.; GRIMM, K. A. Lumb \& Jones: Anestesiologia e analgesia veterinária. 4.ed. São Paulo: ROCA, 2013. p.335-384.

MOHRMANN, D.E.; HELLER, L.J. Cardiovascular physiology. 3.ed. New York: McGraw-Hill, 1997. 224p.

O'BRIEN, P.J.; DAMERON, G.W.; BECK, M.L.; ERICKSONB. K. et al. Cardiac troponin I is a sensitive, specific biomarker of cardiac injury in laboratory animals. Lab. Anim., v.40, p.153-171. 2006.

QUIMBY, J.M; SMITH, M.L; LUNN, K.F. Evaluation of the effects of hospital visit stress on physiologic parameters in the cat. J. Feline Med. Surg., v.13, p.733-737, 2011.

RIVIERE, J.E.; PAPICH, M.G. Veterinary pharmacology and therapeutics. 9.ed. Ames: Wiley-Blackwell, 2009. 1544p.

SAUNDERS A.B.; HANZLICEK, A.S.; MARTINEZ, E.A. et al. Assessment of cardiac troponin I and C-reactive protein concentrations associated with anesthetic protocols using sevoflurane or a combination of fentanyl, midazolam and sevoflurane dogs. Vet. Anaeth. Analg., v.36, p.449-456, 2009.

SHIH, A.C.; MAISENBACHER, V.M.D.; BARREIRINHA, A. et al. Effect of routine cardiovascular catheterization on cardiac troponin I concentration in dogs. J. Vet. Cardiol., v.11, p.587-592, 2009.
SISSON, D. Biochemical markers of cardiac dysfunction. In: WORLD SMALL ANIMAL VETERINARY ASSOCIATION CONGRESS, 27., 2002. Granada, Spain. Proceedings...Granada: WSAVA, 2002.

SLACK, J.; BOSTON, R.; DRIESSEN, B.; REEF, V. Effect of general anesthesia on plasma cardiac troponina I concentrations in healthy horses. J. Vet. Cardiol., v.13, p.163-169, 2011.

SLEEPER, M.M.; CLIFFORD, C.A.; LASTER, L.L. Cardiac troponin I in the normal dog and cat. J. Vet. Intern. Med., v.15, p.501-503, 2001.

SOUZA, A.P.; CARARETO, R.; NUNES, N. et al. Eletrocardiografia em cães anestesiados com Cetamina-S ou Cetamina. Cienc. Rural, v.32, p.787-791, 2002.

TILLEY, P.L. ST segment abnormalities. In: ESSENTIALS of canine and feline eletrocardiography: interpretation and treatment. New York: Lea \& Febiger, 1992. p.84-87.

YONEZAWA, L.A.; SILVEIRA, V.F.; MACHADO, L.P. et al. Marcadores cardíacos na medicina veterinária. Cienc. Rural, v.4, p.222230, 2010.

WARD, J.L.; SCHOBER, K.E.; FUENTES, V.L. Effects of sedation on echocardiographic variables of left atrial and left ventricular function in healthy cats. J. Feline Med. Surg., v.14, p.678-685, 2012.

WELLS, S.M.; SLEEPER, M. Cardiac troponins. J. Vet. Emerg. Crit Care, v.18, p.235-245, 2008.

ZAUGG, M.; SCHAUB, M.C. FOEX, P. Myocardial injury and its prevention in the perioperative setting. Br. J. Anaesth., v.93, p.2133, 2004. 